\title{
Software Defect Prediction Analysis by using Machine Learning Algorithms.
}

\author{
Naveen Babu, Himagiri, V Vamshi Krishna, A Anil Kumar, M Ravi
}

\begin{abstract}
Programming deformation gauge expect a crucial activity in keeping up extraordinary programming and diminishing the cost of programming improvement. It urges adventure executives to relegate time and advantages for desert slanted modules through early flaw distinguishing proof. Programming flaw desire is a matched portrayal issue which orchestrates modules of programming into both 2 arrangements: Defect- slanted and not-deformation slanted modules. Misclassifying blemish slanted modules as not-disfigurement slanted modules prompts a higher misclassification cost than misclassifying not-flaw slanted modules as deformation slanted ones. The AI estimation used in this paper is a mix of Cost-Sensitive Variance Score (CSVS), Cost-Sensitive Laplace Score (CSLS) and Cost-Sensitive Constraint Score (CSCS). The proposed Algorithm is surveyed and demonstrates better execution and low misclassification cost when differentiated and the 3 calculations executed autonomously.
\end{abstract}

Keywords : Cost-Sensitive learning; feature selection; Software defect prediction

\section{INTRODUCTION}

Sdp can be portrayed as a parallel plan issue, where programming modules are assigned either blemish slanted or not-distortion slanted modules, using a great deal of programming estimations. the standard programming estimations include: cyclomatic multifaceted design, halstead unpredict-capacity, number of lines of code ect. most programming flaw desire considers have utilized AI techniques. the underlying advance to build a desire show is to make cases from programming reports, for instance, structure control systems, issue following systems, email accounts, and so on each event can address a system, an item portion, a source code record, a class, a limit, just as a code change as shown by desire granularity. Resulting to making events with estimations and imprints, we can apply pre-dealing with frameworks, which are typical in AI. Pre-getting ready methods used in defect gauge looks at consolidate Feature assurance, Dimension decline, Classification, Prediction finally Performance examination. The stream chart underneath depicts the entire technique of programming distortion conjecture. The true data, including diverse programming estimations got from programming systems, are apportioned into two get-togethers: the planning enlightening gathering, and the test instructive record. These data are preprocessed before being supported into the going with

Revised Version Manuscript Received on 16 September, 2019.

* Correspondence Author

Naveen Babu*, CSE department, JBIET, Hyderabad, India.

Himagiri, CSE department, JBIET, Hyderabad, India.

V Vamshi Krishna, PG Scholar MCA department, JBIET, Hyderabad, India.

A Anil Kumar, PG Scholar MCA department, JBIET, Hyderabad, India.

M Ravi, MCA department, JBIET, Hyderabad, India, ravimjbiet@gmail.com segment assurance and portrayal figurings. In the second stage, cost-sensitive part decision estimations are associated with the planning data to find the perfect features, and as needs be the estimation can be diminished. The consequent stage is to set up the cost-unstable request models subject to the arrangement instructive record with picked features. With the last course of action of planning events, we can set up a desire model. The gauge model can envision whether another event is disfigurement slanted or not-defect slanted.

\section{RELATED WORK}

Numerous examines ponders in 10 years have focused on proposing new estimations to fabricate figure models. For the most part thought about estimations are source code and procedure estimations. Source code estimations measure how source code is eccentric and the estimations are removed from programming reports, for instance, variation control systems and issue following structures that manage all headway stories. Procedure estimations assess various pieces of programming improvement process, for instance, changes of source code, duty regarding code records, planner affiliations, etc. Handiness of procedure estimations for defect desire has been exhibited in various examinations. Most distortion desire ponders are coordinated reliant on truthful technique, for instance AI. Desire models learned by AI figurings can anticipate either bug-tendency of source code (plan) or the amount of disfigurements in source code. Blemish desire models attempted to perceive deserts in structure, fragment/group, or archive/class levels. Progressing examinations exhibited the probability to separate surrenders even in module/method and change levels. Better granularity can help designs by narrowing the degree of source code review for quality insistence. Proposing preprocessing techniques for gauge models is moreover a basic research branch in defect desire contemplates. Prior to building a figure show, we may apply the going with strategies: incorporate decision, institutionalization, and hullabaloo managing. With the preprocessing systems proposed, desire execution could be improved in the related examinations. Researchers also have proposed approaches for cross-adventure defect conjecture. Most agent considers portrayed above have been driven and checked under within figure setting, for instance desire models were collected and attempted in a comparative endeavor. In any case, it is troublesome for new exercises, which don't have enough improvement chronicled information, to fabricate gauge models. Specialist approaches for cross disfigurement desire are metric pay Nearest Neighbor (NN) Filter, Transfer Naive Bayes, These philosophies modify a gauge exhibit by picking similar precedents, changing data regards, or working up another model. 


\section{ENHANCED FEATURE SELECTION}

Highlight decision has been commonly used in numerous model affirmation and AI applications for a significant timeframe. The purpose of feature assurance is to find the unimportantly estimated segment subset that is significant and sufficient for a specific task. The proposed estimation is obtained by including the 3 novel cost tricky counts cost fragile contrast score, cost sensitive laplacian score and cost unstable constraint score to design another condition. The features are first expelled dependent on the 3 counts. The features as such gained are then combined and given as commitment to the proposed computation to make another plan of features. Vacillation score (VS) is a direct unsupervised appraisal proportion of features. It picks features that have the best distinction among all models, with the fundamental imagined that the change among a component space reflects the specialist power of this component. As another pervasive unsupervised part assurance procedure, Laplacian Score (LS) not simply slants toward features with greater variances which have progressively delegate control, yet moreover supports features with more grounded district ensuring limit Constraint Score(CS) is a semi-regulated incorporate decision technique, which performs incorporate assurance as shown by the impediment sparing limit of features It uses must-interface and can't associate match astute necessities as supervision information, where incorporates that can best secure the must-associate goals similarly as the can't associate objectives are believed to be basic. Existing part assurance techniques proposed for SDP are cost-stupor, i.e., the issue of different costs for different botches isn't considered. The proposed computation is procured by including the 3 novel cost sensitive counts cost fragile vacillation score, cost unstable laplacian score and cost tricky prerequisite score to figure another condition. The features are first isolated dependent on the 3 estimations. The features along these lines got are then merged together and given as commitment to the proposed count to create another plan of features.[4] The educational accumulations used in this examination begin from individuals as a rule NASA Metrics Data Program (MDP) store. These educational accumulations, including CM1, $\mathrm{KC} 2, \mathrm{MW} 1, \mathrm{PC} 1, \mathrm{PC} 2, \mathrm{PC} 3$, and PC4, have a spot with a couple of NASA adventures. The extent of execution of an AI figuring relies upon its precision of masterminding an instructive record. In most evident applications; different misclassifications are regularly associated with different costs. [2]Denote class checks as $\{1 \ldots \mathrm{c}\}$. Misclassifying a precedent from the ith class as the cth class will cause more noteworthy costs than misclassifying a case of the cth class as various classes. Here, we call the class from the top notch to the (c-1)th class the in-store up class, while the cth class is known as the out-total class. By then, we can mastermind misclassification costs into three sorts:

1) the cost of false affirmation, i.e., the cost of misclassifying a model from the out-amass class as being from the in-bundle class;

2) the cost of false expulsion, i.e., the cost of misclassifying a model from the in-bundle class as being from the out-gather class; and
3) the cost of false conspicuous verification, i.e., the cost of misclassifying a model from one in-gather class as being from another in-cluster class.

To address the differentiating cost of each kind of misclassification, a cost framework given underneath can be used.[5]

\begin{tabular}{|l|l|l|}
\hline & Defect-prone & Not-defect-prone \\
\hline Defect -prone & $\mathrm{C}(0,0)=\mathrm{c}_{00}$ & $\mathrm{C}(0,1)=\mathrm{c}_{01}$ \\
\hline Not-defect-prone & $\mathrm{C}(1,0)=\mathrm{c}_{10}$ & $\mathrm{C}(1,1)=\mathrm{c}_{11}$ \\
\hline
\end{tabular}

Fig 1:Cost Matrix

IV. Prediction

The features evacuated using improved component assurance are used in the test data and checked if the regard falls inside the range. The delayed consequence of desire can be imparted as a perplexity arrange show up underneath.

Real positive(TP) : defect slanted module foreseen as deformation slanted.

False positives(FP) : not-defect slanted module foreseen as deformation slanted.

Real negative(TN) : not-defect slanted module foreseen as not-deformation slanted module.

False negative(FN) : defect slanted module foreseen as not-deformation slanted.

\begin{tabular}{|l|l|l|}
\hline & Defect-prone & Not-defect-prone \\
\hline Defect -prone & TP & FN \\
\hline Not-defect-prone & FP & TN \\
\hline
\end{tabular}

Fig 2:Confusion matrix

\section{EXPERIMENTS RESULTS}

For better surveying the shows in the cost-sensitive learning circumstances, the Total-cost of misclassification, which is a general estimation for cost-unstable learning, is used as one fundamental evaluation standard in our preliminaries. Of course, as showed up in Fig 2, the portrayal results can be addressed by the disorder structure with two lines and two areas reporting the amount of authentic positives, false positives, false negatives, and real negatives.

$$
\text { Sensitivity }=\frac{\mathrm{TP}}{\mathrm{TP}+\mathrm{FN}}
$$

$$
\text { Accuracy }=\frac{(\mathrm{TP}+\mathrm{TN})}{(\mathrm{TP}+\mathrm{FN}+\mathrm{FP}+\mathrm{TN})}
$$

Where affectability evaluates the degree of distortion slanted modules precisely requested, and precision gauges the degree of tests successfully described among the whole people. Despite the Total-cost, we in like manner grasp the affectability and accuracy of the request results as appraisal measures. 


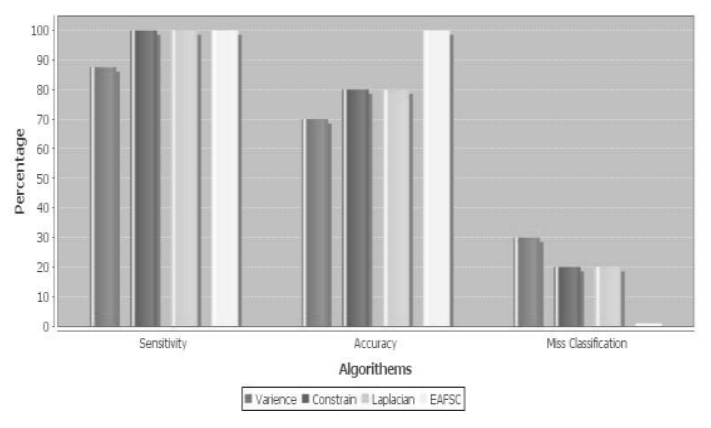

Fig 3: Comparison of different feature selection algorithms One can see that the improved estimation has much lower misclassification cost than CSVS,CSLS,CSCS. It in like manner has better precision when appeared differently in relation to the next 3 counts. The Sensitivity is similarly equivalent to the following three counts.

\section{Conclusion}

The updated estimation is gained by including 3 cost-fragile computations to be explicit Cost-Sensitive Variance Score (CSVS), Cost-Sensitive Laplace Score (CSLS) and Cost-Sensitive Constraint Score (CSCS).From tests the dataset assembled from NASA, its saw that the improved count makes ideal execution over when the 3 figuring's are executed freely..

\section{REFERENCES}

1. I. Guyon, S. Gunn, M. Nikravesh, and Z. L., Feature

Extraction:Foundations and Applications. Berlin, Germany: Springer-VerlagNew York,Inc., 2006.

2. D. Sun and D. Zhang, "Bagging constraint score for feature selection with pairwise constraints," Pattern Recogn., vol. 43, pp. 2106-2118,2010.

3. S. Kim, Z. T. J. Whitehead, and A. Zeller, "Predicting faults from cached history," in Proc. 29th Int. Conf. Software Eng., Washington, DC,USA, 2007, pp. 489-498.

4. C. Seiffert, T. M. Khoshgoftaar, J. V. Hulse, and A. Napolitano, "A comparative study of data sampling and cost sensitive learning," in Proc.IEEE Int. Conf. DataMiningWorkshops, Washington, DC, USA, 2008, pp. 46-52.

5. Z.-H. Zhou and X.-Y. Liu, "On multi-class cost-sensitive learning," in Proc. 21st National Conf. Artificial Intelligence, 2006,pp.567-572.

6. A. Bernstein, J. Ekanayake, andM. Pinzger, "Improving defect prediction using temporal features and non linear models," in 9th Int. WorkshoponPrinciples of Software Evolution, Dubrovnik, Croatia, 2007, pp. 11-18.

7. Y. Bo and L. Xiang, "A study on software reliability prediction based on support vector machines," in Proc. IEEE Int. Conf. Ind. Eng.Eng.Manag., Singapore, 2007, pp. 1176-1180.

8. L. Guo, Y. Ma, B. Cukic, and H. Singh, "Robust prediction of defect proneness by random forests," in Proc. 15th Int. Symp. Software Rel.Eng.,2010.

9. Mingxia Liu, LinsongMiao, and Daoqiang Zhang, "Two-Stage Cost-Sensitive Learning for Software Defect Prediction," IEEE Transactions onreliability, Vol. 63, No. 2, June 2014.

10. P. D. Turney, "Cost-sensitive classification: Empirical evaluation of a hybrid genetic decision tree induction algorithm," J. Artif. Intell. Res., vol.2, pp. 369-409,2011.

11. R. Moser, W. Pedrycz, and G. Succi, "A comparative analysis of the efficiency of change metrics and static code attributes for defectprediction," in Proc. 30th Int. Conf. Software Eng., Leipzig, Germany, 2008, pp. 181-190.

\section{AUTHORS PROFILE}

NAVEEN BABU*, CSE department, JBIET, Hyderabad, India.

HIMAGIRI, CSE department, JBIET, Hyderabad, India.

V VAMSHI KRISHNA, PG Scholar MCA department, JBIET, Hyderabad, India.

A ANIL KUMAR PG Scholar MCA department, JBIET, Hyderabad, India

M RAVI, MCA

ravimjbiet@gmail.com department, JBIET, Hyderabad, India, 